\title{
Black Sun: Ocular Invisibility of Relativistic Luminous Astrophysical Bodies
}

\author{
Jeffrey S. Lee ${ }^{1}$, Gerald B. Cleaver ${ }^{1,2}$ \\ ${ }^{1}$ Early Universe Cosmology and Strings Group, Center for Astrophysics, Space Physics, and Engineering Research, Waco, TX, USA \\ ${ }^{2}$ Department of Physics, Baylor University, Waco, TX, USA \\ Email: Jeff_Lee@Baylor.edu, Gerald_Cleaver@Baylor.edu
}

How to cite this paper: Lee, J.S. and Cleaver, G.B. (2016) Black Sun: Ocular Invisibility of Relativistic Luminous Astrophysical Bodies. Journal of High Energy Physics, Gravitation and Cosmology, 2, 562-570.

http://dx.doi.org/10.4236/jhepgc.2016.24048

Received: July 26, 2016

Accepted: September 9, 2016

Published: September 12, 2016

Copyright $\odot 2016$ by authors and Scientific Research Publishing Inc. This work is licensed under the Creative Commons Attribution International License (CC BY 4.0).

http://creativecommons.org/licenses/by/4.0/

\section{(c) (i) Open Access}

\begin{abstract}
Considered as a gedanken experiment are the conditions under which the relativistic Doppler shifting of visible electromagnetic radiation to beyond the human ocular range could reduce the incident radiance of the source, and render a luminous astrophysical body (LAB) invisible to a naked eye. This paper determines the proper distance as a function of relativistic velocity at which a luminous object attains ocular invisibility.
\end{abstract}

\section{Keywords}

Relativistic Processes, Black Hole Physics, Reference Systems, Luminous

Astrophysical Body, Relativistic Blackbody Spectrum

\section{Introduction}

The relativistic blackbody spectrum [1] suggests the intriguing possibility that a luminous astrophysical body can be rendered optically invisible to the human eye by relativistic Doppler shifting the wavelengths of maximum intensity from the visible frequency range to above or below the frequency thresholds of human vision. ${ }^{1}$ In honor of the $100^{\text {th }}$ anniversary of Einstein's general relativity, this note examines, as a gedanken experiment, the specific conditions under which this effect would occur. ${ }^{2}$

Furthermore, relativistic blackbody radiators will emit spectral radiances which are increased (in the case of approaching) or decreased (in the case of receding), due to temperature inflation and relativistic beaming. By considering in the gedanken experi-

${ }^{1}$ For this effect, CCD detectors could be considered in place of human eyes, but the nature of the phenomenon wouldn't change. Of course, the numbers would strongly change, due to the strong differences between the spectral response function of human eye and CCD cameras: for instance, a relativistic Doppler shift could make the object even more detectable by an IR sensitive CCD. 
ment the relativistic blackbody spectrum, the proper distances can be determined at which the apparent magnitude of a blackbody radiator is greater (i.e. dimmer) than approximately 6.5 (the threshold of vision for the typical unaided human eye).

Additionally, laboratory tests of the sensitivity of the unassisted human eye are described, and this paper asserts that the Judd \& Voss CIE 1978 photopic luminous efficiency function would not be applicable to the situation of LABs due to the much greater luminosity than in the laboratory tests.

\section{The Apparent Magnitude of Blackbody Radiators in the Rest Frame}

The relationship between absolute magnitude, apparent magnitude, and distance to an arbitrary stationary blackbody radiation source has been well established and is given by:

$$
M=m+5-5 \log z
$$

where $M$ is the absolute magnitude of any blackbody radiator, $m$ is its apparent magnitude, and $z$ is the distance to the observer in parsecs. Also, in terms of luminosity,

$$
M=M_{o}-2.5 \log \left(\frac{L}{L_{o}}\right)
$$

where $M_{o}$ is the absolute magnitude of a reference star (e.g. the sun), $L$ is the luminosity of the radiation source at an arbitrary distance $z$, and $L_{o}$ is the absolute luminosity of that source ${ }^{3}$.

Equating Equations (1) and (2) yields:

$$
Z=10 \frac{m+5-M_{o}}{5} \sqrt{\frac{L}{L_{o}}} .
$$

Thus, for the sun, $M_{o}=4.83, L=L_{o}=1$, and for it to be invisible to the naked eye in the [nearly] total blackness of interstellar space, $m=6.5$ [2] (discussed in Section 4). Therefore, the sun is visible to the unaided eye at distances up to $21.58 \mathrm{pc}(70.39 \mathrm{LY})$.

\section{The Apparent Magnitude of Relativistic Blackbody Radiators}

Sufficiently high speed relativistic motion of blackbody radiators would clearly Doppler shift the wavelengths of maximum luminosity to beyond the human visual range. Therefore, the lower luminosity wavelengths are Doppler shifted into the visible range, and the overall visible luminosity is reduced.

However, in the case of an approaching blackbody, the radiation is relativistically beamed, and the blackbody temperature is "inflated". Both of these effects serve to in-

${ }^{2}$ Whether the physical situations could exist for this effect to be realized is VERY uncertain. As an example, relativistic speeds might be obtainable in the expulsion of a low-mass star from the region of the Galactic Center as a consequence of a fly-by with the central massive black hole. Nevertheless, such a star might already appear invisible from earth because of its distance, rather than as a result the relativistic Doppler Effect and would be strongly decelerated (or even completely destroyed) by the relativistic drag of the dense interstellar matter of the Galactic Center region. The possible astrophysical application of this gedanken experiment is thus unlikely.

${ }^{3}$ The luminosity of the source at the absolute magnitude distance (i.e., 10 parsecs). 
crease the luminosity. For a receding blackbody, relativistic beaming ("expanding") and temperature "deflation" will have the reverse effect. Therefore, Equation (3) becomes:

$$
z=10^{\frac{m+5-M_{o}}{5}} \sqrt{\frac{L^{\prime}}{L_{o}}}
$$

where $L^{\prime}$ and $L_{o}$ are the luminosities in the relativistic and rest frames respectively ${ }^{4}$.

Luminosity is obtained by integrating the spectral radiance over frequency and solid angle:

$$
\frac{L^{\prime}}{L_{o}}=\frac{\int_{v_{1}}^{v_{2}} \int B^{\prime} \mathrm{d} \Omega^{\prime} \mathrm{d} v^{\prime}}{\int_{v_{1}}^{v_{2}} \int B_{o} \mathrm{~d} \Omega \mathrm{d} v}
$$

where $v_{1}$ and $v_{2}$ are the mean lower and upper frequencies of ocular visibility. $B^{\prime}$ and $B_{o}$ are the spectral radiances in the relativistic and rest frames respectively, which must be integrated over the appropriate solid angle $\Omega$. The relativistic spectral radiance in frequency space, accounting for Doppler shifting, relativistic beaming, and temperature inflation, was determined by Lee and Cleaver [1], and is given by ${ }^{5}$ :

$$
B_{v}^{\prime} \mathrm{d} \Omega=\int_{v_{1}}^{v_{2}} \frac{\left(\frac{2 h v^{3}}{c^{2}}\right)}{\exp \left[\frac{h v}{k_{B}}\left(\beta_{t}-\beta_{z} \cos \theta\right)\right]-1}[\gamma(1-V \cos \theta)]^{-3} \mathrm{~d} v \mathrm{~d} \Omega
$$

where [3],

$$
\begin{aligned}
& \beta_{t}=\frac{1}{T_{o} \sqrt{1-V^{2}}} \\
& \beta_{z}=\frac{V}{T_{o} \sqrt{1-V^{2}}}
\end{aligned}
$$

and $T_{o}$ is the proper absolute temperature, $u_{\mu}$ is the relative 4-velocity between the radiation and the observer, $\beta_{\mu}=\beta_{t}-\beta_{z} \cos \theta$ is the van Kampen-Israel inverse temperature 4-vector, $\theta$ is the angle between $u_{\mu}$ and $\beta_{\mu}, V=\frac{u}{c}$ (fraction of light speed).

The integration of the spectral radiance over all frequencies is straightforward because, with the limits of 0 and $\infty$, the result is simply $\pi^{4} / 15$. However, the in-band luminosity requires integration over a finite frequency range. Here, the method of Widger and Woodall is followed [4].

$$
B_{v}^{\prime} \mathrm{d} \Omega=\int_{v_{1}}^{v_{2}} \frac{\left(\frac{2 h v^{3}}{c^{2}}\right)}{\exp \left[\frac{h v}{k_{B}}\left(\beta_{t}-\beta_{z} \cos \theta\right)\right]-1}[\gamma(1-V \cos \theta)]^{-3} \mathrm{~d} v \mathrm{~d} \Omega
$$

${ }^{4}$ Primed quantities indicate the relativistic frame.

${ }^{5}$ For a more detailed discussion of the effects of temperature inflation and relativistic beaming on spectral radiance, see [1]. 
Letting:

$$
Q^{\prime}=\frac{\left(\frac{2 h}{c^{2}}\right)\left(1-V^{2}\right)^{\frac{3}{2}}}{(1-V \cos \theta)^{3}}
$$

and

$$
R^{\prime}=\frac{h(1-V \cos \theta)}{k_{B} T_{o} \sqrt{1-V^{2}}} .
$$

Also, letting $x^{\prime}=R^{\prime} v$, and from Equations (7) and (8), Equation (9) becomes:

$$
B_{v}^{\prime} \mathrm{d} \Omega=\frac{Q^{\prime}}{R^{\prime}} \int_{v_{1}^{\prime}}^{v_{2}^{\prime}} \frac{x^{\prime 3}}{{x^{\prime}}^{\prime}-1} \mathrm{~d} x^{\prime} \mathrm{d} \Omega .
$$

Expanding Equation (12) as a difference of integrals:

$$
B_{v}^{\prime} \mathrm{d} \Omega=\frac{Q^{\prime}}{R^{\prime 4}}\left[\int_{\frac{x_{1}^{\prime}}{R}}^{\infty} \frac{x^{\prime 3}}{\mathrm{e}^{x^{\prime}}-1}-\int_{\frac{x_{2}^{\prime}}{R}}^{\infty} \frac{x^{\prime 3}}{\mathrm{e}^{x^{\prime}}-1}\right] \mathrm{d} x^{\prime} \mathrm{d} \Omega .
$$

Evaluating Equation (13), and re-substituting $x^{\prime}=R^{\prime} v$ :

$$
\begin{aligned}
B_{v}^{\prime} \mathrm{d} \Omega=\frac{Q^{\prime}}{R^{\prime}} & {\left[\sum_{n=1}^{\infty}\left(\frac{R^{\prime 3} v_{1}^{3}}{n}+\frac{3 R^{\prime 2} v_{1}^{2}}{n^{2}}+\frac{6 R^{\prime} v_{1}}{n^{3}}+\frac{6}{n^{4}}\right) \mathrm{e}^{-n R^{\prime} v_{1}}\right.} \\
& \left.-\sum_{n=1}^{\infty}\left(\frac{R^{\prime 3} v_{2}^{3}}{n}+\frac{3 R^{\prime 2} v_{2}^{2}}{n^{2}}+\frac{6 R^{\prime} v_{2}}{n^{3}}+\frac{6}{n^{4}}\right) \mathrm{e}^{-n R^{\prime} v_{2}}\right] \mathrm{d} \Omega
\end{aligned}
$$

Expanding the solid angle integration, combining sums, and making use of $L_{v}^{\prime}=\int_{v_{1}}^{v_{2}} \int B^{\prime} \mathrm{d} \Omega^{\prime} \mathrm{d} v^{\prime}$ from Equation (5), the relativistic luminosity in frequency space (Equation (14)) becomes ${ }^{6}$ :

$$
\begin{aligned}
L_{v}^{\prime}=\frac{Q^{\prime}}{R^{\prime}} \iint \sum_{n=1}^{\infty} & {\left[\left(\frac{R^{\prime 3} v_{1}^{3}}{n}+\frac{3 R^{\prime 2} v_{1}^{2}}{n^{2}}+\frac{6 R^{\prime} v_{1}}{n^{3}}+\frac{6}{n^{4}}\right) \mathrm{e}^{-n R^{\prime} v_{1}}\right.} \\
& \left.-\left(\frac{R^{\prime 3} v_{2}^{3}}{n}+\frac{3 R^{\prime 2} v_{2}^{2}}{n^{2}}+\frac{6 R^{\prime} v_{2}}{n^{3}}+\frac{6}{n^{4}}\right) \mathrm{e}^{-n R^{\prime} v_{2}}\right] \cos \theta \sin \theta \mathrm{d} \theta \mathrm{d} \phi
\end{aligned}
$$

In the case of approaching the LAB [approximately] directly, a simplification of Equation (15), which cannot be resolved as a closed form function, can be made. Since, $\theta$ is very small $\sin \theta \sim \theta$ and $\cos \theta \sim 1$. This removes the angular dependence from Equation (11), which reduces to:

$$
R=\frac{h}{k_{B} T_{o}} \sqrt{\frac{1-V}{1+V}} .
$$

Frequently, when evaluating the $d \Omega$ integration, the solid angle over which the inte${ }^{6}$ The $\cos \theta$ term accounts for the Lambertian radiator, and the $\sin \theta$ term arises from the solid angle integration. 
gration is performed is the solid angle through which the blackbody radiates. However, that is not the case here. The solid angle is that which is subtended by the blackbody from the vantage point of the observer. Therefore, when $z \gg D, \theta \sim \frac{D}{z}$ ( $z$ is the observer proper distance, and $D$ is the diameter of the blackbody). For a blackbody with a circular $x$ - $y$ cross-section, $\phi \sim \frac{D}{Z}$. Therefore,

$$
\begin{aligned}
L_{v}^{\prime} \approx \frac{Q^{\prime}}{R^{\prime 4}} \sum_{n=1}^{\infty} & {\left[\left(\frac{R^{\prime 3} v_{1}^{3}}{n}+\frac{3 R^{\prime 2} v_{1}^{2}}{n^{2}}+\frac{6 R^{\prime} v_{1}}{n^{3}}+\frac{6}{n^{4}}\right) \mathrm{e}^{-n R^{\prime} v_{1}}\right.} \\
& \left.-\left(\frac{R^{\prime 3} v_{2}^{3}}{n}+\frac{3 R^{\prime 2} v_{2}^{2}}{n^{2}}+\frac{6 R^{\prime} v_{2}}{n^{3}}+\frac{6}{n^{4}}\right) \mathrm{e}^{-n R^{\prime} v_{2}}\right]_{0}^{\frac{D}{2}} \theta \mathrm{d} \theta \int_{0}^{\frac{D}{2}} \mathrm{~d} \phi .
\end{aligned}
$$

Thus,

$$
\begin{aligned}
L_{v}^{\prime} \approx \frac{2 k_{B}^{4} T_{o}^{4}}{c^{2} h^{3}} \sum_{n=1}^{\infty} & {\left[\left(\frac{R^{\prime 3} v_{1}^{3}}{n}+\frac{3 R^{\prime 2} v_{1}^{2}}{n^{2}}+\frac{6 R^{\prime} v_{1}}{n^{3}}+\frac{6}{n^{4}}\right) \mathrm{e}^{-n R^{\prime} v_{1}}\right.} \\
& \left.-\left(\frac{R^{\prime 3} v_{2}^{3}}{n}+\frac{3 R^{\prime 2} v_{2}^{2}}{n^{2}}+\frac{6 R^{\prime} v_{2}}{n^{3}}+\frac{6}{n^{4}}\right) \mathrm{e}^{-n R^{\prime} v_{2}}\right]\left(\frac{D}{z}\right)^{3}\left(\frac{1-V}{1+V}\right)^{\frac{7}{2}} .
\end{aligned}
$$

Similarly,

$$
\begin{aligned}
L_{o} \approx \frac{2 k_{B}^{4} T_{o}^{4}}{c^{2} h^{3}} \sum_{n=1}^{\infty} & {\left[\left(\frac{R^{3} v_{1}^{3}}{n}+\frac{3 R^{2} v_{1}^{2}}{n^{2}}+\frac{6 R v_{1}}{n^{3}}+\frac{6}{n^{4}}\right) \mathrm{e}^{-n R v_{1}}\right.} \\
& \left.-\left(\frac{R^{3} v_{2}^{3}}{n}+\frac{3 R^{2} v_{2}^{2}}{n^{2}}+\frac{6 R v_{2}}{n^{3}}+\frac{6}{n^{4}}\right) \mathrm{e}^{-n R v_{2}}\right]\left(\frac{D}{z}\right)^{3} .
\end{aligned}
$$

Combining Equation (3), in terms of relativistic luminosity, with Equations (18) and (19) yields:

$$
\begin{aligned}
& Z=10 \frac{m+5-M_{o}}{5} \\
& {\left[\frac{\sum_{n=1}^{\infty}\left[\left(\frac{R^{\prime 3} v_{1}^{3}}{n}+\frac{3 R^{\prime 2} v_{1}^{2}}{n^{2}}+\frac{6 R^{\prime} v_{1}}{n^{3}}+\frac{6}{n^{4}}\right) \mathrm{e}^{-n R^{\prime} v_{1}}-\left(\frac{R^{\prime 3} v_{2}^{3}}{n}+\frac{3 R^{\prime 2} v_{2}^{2}}{n^{2}}+\frac{6 R^{\prime} v_{2}}{n^{3}}+\frac{6}{n^{4}}\right) \mathrm{e}^{-n R^{\prime} v_{2}}\right]}{\sum_{n=1}^{\infty}\left[\left(\frac{R^{3} v_{1}^{3}}{n}+\frac{3 R^{2} v_{1}^{2}}{n^{2}}+\frac{6 R v_{1}}{n^{3}}+\frac{6}{n^{4}}\right) \mathrm{e}^{-n R v_{1}}-\left(\frac{R^{3} v_{2}^{3}}{n}+\frac{3 R^{2} v_{2}^{2}}{n^{2}}+\frac{6 R v_{2}}{n^{3}}+\frac{6}{n^{4}}\right) \mathrm{e}^{-n R v_{2}}\right]}\right]^{\frac{1}{2}}\left(\frac{1-V}{1+V}\right)^{\frac{7}{4}}(20)}
\end{aligned}
$$

Evaluation of the infinite sums is greatly simplified due, in large part, to the rapid convergence of the series as a result of the $\mathrm{e}^{-n R v}$ terms. The smallest value of $R$ (requiring the largest number of summation terms) occurs when $V=0$, and from Equation (11), is $\frac{h}{k_{B} T_{o}}$. The smallest useful value of $R v=0.400$ would result from an Oclass star, with a surface temperature of $\sim 50,000 \mathrm{~K}$, and at the lowest frequency of human visibility. Table 1 gives the number of summation terms (n) (in Equation (20)) that would be required to produce at least 10 significant figure convergence for $0.1 \leq R v \leq 25$. 
Table 1. Number of summation terms required for series convergence of Equation (20) to at least 10 significant figures [4].

\begin{tabular}{cc}
\hline$R v$ & Number of summation terms $(n)$ \\
\hline 0.1 & 101 \\
0.2 & 65 \\
0.3 & 50 \\
0.4 & 50 \\
0.5 & 35 \\
0.6 & 30 \\
0.7 & 25 \\
0.8 & 22 \\
$0.9-1.4$ & 20 \\
$1.5-1.9$ & 15 \\
$2.0-2.9$ & 10 \\
$3.0-3.9$ & 8 \\
$4.0-4.9$ & 6 \\
$5.0-9.9$ & 4 \\
$10.0-24.9$ & 3 \\
$\geq 25.0$ & 1 \\
\hline
\end{tabular}

\section{The Ocular Invisibility of Relativistic Radiators}

The visibility to the naked eye of astronomical objects has been discussed extensively in the literature [5]-[9]. The "sky" of interstellar space is considered to be absolutely black, and a viewing port is taken to be, at optical wavelengths, a perfectly transparent aperture that subtends a solid angle of at least the human field of vision and with a magnification of 1.

The efficiency by which photons are used by the retina was accounted for by correcting for the Stiles-Crawford effect of the first (SCE I) and second (SCE II) kind ${ }^{7}$, photon absorption by the optical media, photopigment absorption of photons, and the photon isomerization efficiency of the photopigment.

For a $22^{\prime}$ (diameter), $10 \mathrm{~ms}, 507 \mathrm{~nm}$ monochromatic source, in which, of the $\sim 100$ quanta incident upon the retina, 10 to 15 were absorbed by the $\sim 1600$ illuminated rods [10]. From this experiment, Packer and Williams determined the rod actinometric, radiometric, and photometric ${ }^{8}$ absolute thresholds to be $0.35 \mathrm{\gamma} / \mathrm{s}, 4.35 \times 10^{-6} \mathrm{~W} \cdot \mathrm{m}^{-2} \cdot \mathrm{sr}^{-1}$, and $1.33 \times 10^{-3} \mathrm{~cd} \cdot \mathrm{m}^{-2}$ respectively [11]. However, also examined was the case of a stimulus which exceeded the visual system's spatial summation area and temporal inte${ }^{7}$ The Stiles-Crawford effect of the first kind is the phenomenon by which light entering the edge of the pupil elicits a smaller response from the cone photoreceptors than light entering the center of the pupil. The Stiles-Crawford effect of the second kind states that the perceived hue from a monochromatic light source is dependent on its obliquity with respect to the retina.

${ }^{8}$ Assuming a $6 \mathrm{~mm}$ in diameter pupil. 
gration time, in which the rod actinometric, radiometric, and photometric absolute thresholds are $2.00 \times 10^{-4} \mathrm{\gamma} / \mathrm{s}, 2.47 \times 10^{-9} \mathrm{~W} \cdot \mathrm{m}^{-2} \cdot \mathrm{sr}^{-1}$, and $7.5 \times 10^{-7} \mathrm{~cd} \cdot \mathrm{m}^{-2}$ respectively.

However, even accounting for the standard observer's spectral sensitivity by applying the Judd \& Voss CIE 1978 photopic luminous efficiency function, these results are difficult to apply to the scenario presented here because of the enormous disparity between the spectral irradiances of the Hallett test sources [10] and stars.

The frequency range of human vision is slightly variable. However, $4.17 \times 10^{14} \mathrm{~Hz}$ and $7.89 \times 10^{14} \mathrm{~Hz}$, which correspond to wavelengths of $720 \mathrm{~nm}$ and $380 \mathrm{~nm}$ respectively, are acceptable approximations of the limits of human vision, and are in keeping with the wavelengths of $700 \mathrm{~nm}$ and $390 \mathrm{~nm}$ published by Starr [12]. The limiting magnitude of the unassisted human eye is taken to be 6.5 [2]. This figure applies to all visible wavelengths and accounts for eye sensitivity. Consequently, inclusion of the Judd \& Voss CIE 1978 photopic luminous efficiency function would not be appropriate.

\section{1. $\theta=0$ Ocular Invisibility}

In the case of approaching the sun directly $(\theta=0)$, the distance at which the apparent magnitude is 6.5 can be determined from Equation (20), and is shown in Figure 1.

\subsection{Ocular Invisibility for Arbitrary $\theta$}

In order to determine the ocular invisibility curve for an arbitrary velocity vector, the solid angle integration in (15), and correspondingly for the stationary case, must be performed. However, since the solid angle over which the integration must be taken does not significantly exceed $\sim 10 \operatorname{mrad}^{9}$ (and is considered primarily for angles much smaller), $z$ can be approximated as being constant at each value of $\theta$ in the 315 time step iterative scheme, which was used to evaluate the solid angle integral. When Equation (20) is evaluated for the sun, Figure 2 results.

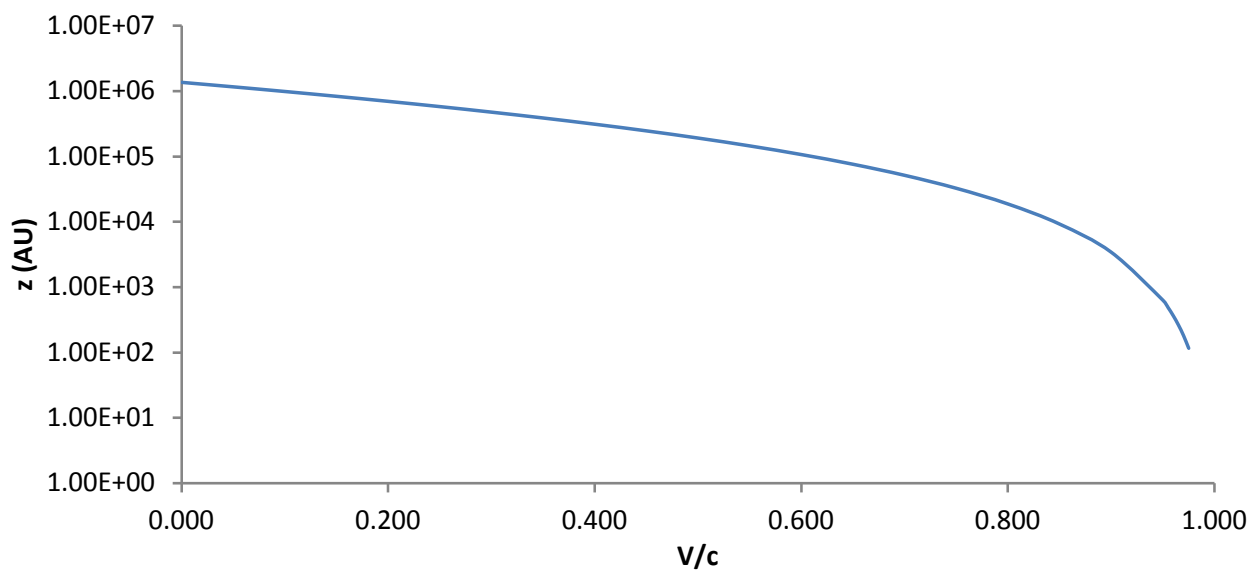

Figure 1. Distance versus speed for limiting magnitude $(m=6.5)$ of the sun. The wavelengths of vision are taken to be between $380 \mathrm{~nm}$ and $720 \mathrm{~nm}$, and the temperature is $5780 \mathrm{~K}$. The region below the curve represents the distance at which the sun is visible to the typical unaided eye of an observer in the frame of the sun.

${ }^{9}$ The angle subtended by the sun at approximately $1 \mathrm{AU}$. 


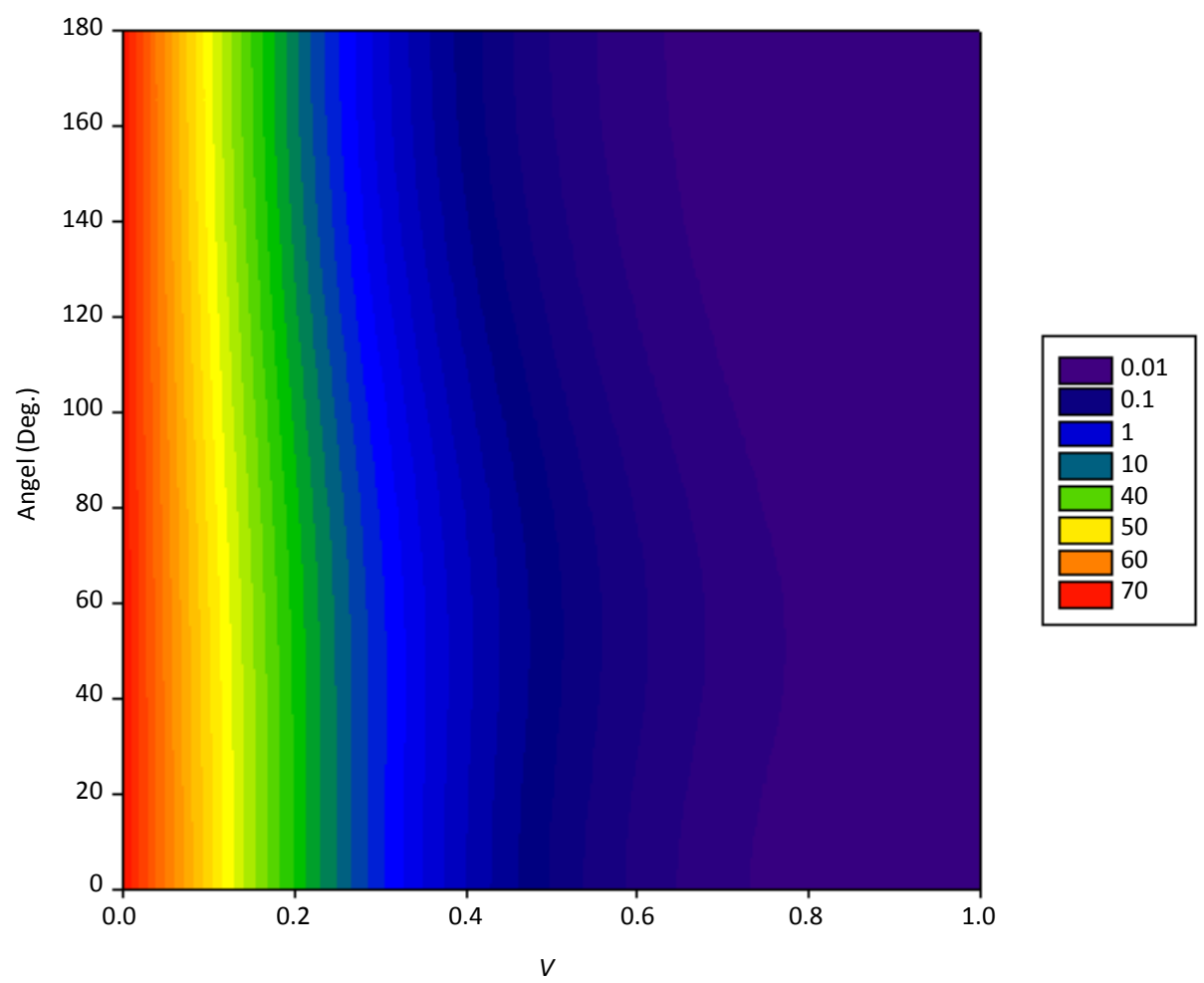

Figure 2. Proper distance of limiting magnitude as a function of $V$ and $\theta$ for the sun. The wavelengths of vision are taken to be $380 \mathrm{~nm}$ to $720 \mathrm{~nm}$, and the temperature is $5780 \mathrm{~K}$. The numbers in the legend represent the proper distances at which the apparent magnitude is 6.5 . The purple region of $0.01 \mathrm{LY}$ represents proper distances which are $\leq 0.01 \mathrm{LY}$. The intersection of the contours with the Angle-axis is expectedly $70.39 \mathrm{LY}$ (as determined in Section 2).

As expected, and shown in Figure 2, ultra-relativistic velocities permit exceptionally close approaches to luminous astrophysical bodies, while maintaining an apparent magnitude which is less than the limiting magnitude of the unaided human eye.

\section{Conclusions}

By making use in this gedanken experiment of the relativistic blackbody spectrum, the velocity profile for the apparent magnitude of a LAB has been determined. Optical invisibility to the unaided eye arises due to the Doppler shifting of the wavelengths of maximum radiance to beyond the limits of human visual sensitivity. Temperature inflation and relativistic beaming can either increase this incident radiance (for an approaching source) or decrease it (for a receding source). By considering the wavelength limits of human vision to be $380 \mathrm{~nm}$ and $720 \mathrm{~nm}$, and the limiting magnitude of the unaided human eye to be 6.5 , the proper distance versus velocity function for ocular invisibility of relativistic luminous astrophysical bodies has been determined; this profile was determined for the sun.

Whether the physical situations could exist for this effect to be realized is uncertain. As an example, relativistic speeds might be obtainable in the expulsion of a low-mass star from the region of the galactic center as a consequence of a fly-by with the central 
massive blackhole. Nevertheless, such a star might already appear invisible from earth because of its distance, rather than as a result the relativistic Doppler Effect. The possible realization of this gedanken experiment is an open question.

\section{References}

[1] Lee, J.S. and Cleaver, G.B. (2015) The Relativistic Blackbody Spectrum in Inertial and NonInertial Reference Frames. BU-HEPP-15-04, CASPER-15-01.

[2] Weaver, H. (1947) The Visibility of Stars without Optical Aid. Publications of the Astronomical Society of the Pacific, 59, 233. http://dx.doi.org/10.1086/125956

[3] de Parga, G.A., Vargas, A.Á. and López-Carrera, B. (2013) On a Self-Consistency Thermodynamical Criterion for Equations of the State of Gases in Relativistic Frames. Entropy, 15, 1271-1288. http://dx.doi.org/10.3390/e15041271

[4] Widger, W. and Woodall, M. (1976) Integration of the Planck Blackbody Radiation Function. Bulletin of the American Meteorological Society, 57, 1217-1219. http://dx.doi.org/10.1175/1520-0477(1976)057<1217:IOTPBR>2.0.CO;2

[5] Weaver, H.F. (1947) The Visibility of Stars without Optical Aid. Publications of the Astronomical Society of the Pacific, 59, 232-243. http://dx.doi.org/10.1086/125956

[6] Johnson, E.P. and Bartlett, N.R. (1956) Effect of Stimulus Duration on Electrical Responses of the Human Retina. Journal of the Optical Society of America, 46, 167-170. http://dx.doi.org/10.1364/JOSA.46.000167

[7] Schaefer, B.E. (1993) Astronomy and the Limits of Vision. Vistas in Astronomy, 36, 311361. http://dx.doi.org/10.1016/0083-6656(93)90113-X

[8] Hughes, D. (1983) On Seeing Stars Especially up Chimneys. Quarterly Journal of the Royal Astronomical Society, 24, 246-257.

[9] Schaefer, B.E. (1991) Glare and Celestial Visibility. Publications of the Astronomical Society of the Pacific, 103, 645-660. http://dx.doi.org/10.1086/132865

[10] Hallett, P. (1987) Quantum Efficiency of Dark-Adapted Human Vision. Journal of the Optical Society of America A, 4, 2330-2335. http://dx.doi.org/10.1364/JOSAA.4.002330

[11] Packer, O. and Williams, D.R. (2003) The Science of Color. In: Shevell, S.K., Ed., Optical Society of America, Kidlington Oxford.

[12] Starr, C., Evers, C.A. and Starr, L. (2006) Biology: Concepts and Applications. Thomson Brooks/Cole. 
Submit or recommend next manuscript to SCIRP and we will provide best service for you:

Accepting pre-submission inquiries through Email, Facebook, LinkedIn, Twitter, etc. A wide selection of journals (inclusive of 9 subjects, more than 200 journals)

Providing 24-hour high-quality service

User-friendly online submission system

Fair and swift peer-review system

Efficient typesetting and proofreading procedure

Display of the result of downloads and visits, as well as the number of cited articles

Maximum dissemination of your research work

Submit your manuscript at: http://papersubmission.scirp.org/ 